\title{
Abstracts from the 2019 International Massage Therapy Research Conference
}

\section{COMPETENCIES DEVELOPED FOR HOSPITAL BASED MASSAGE THERAPY}

M.K. Brennan, MS, RN, LMBT, Dale Healey DC, $\mathrm{PhD}$, Carolyn Tague MA, CMT, Beth Rosenthal PhD, MBA, MPH

Introduction: As massage therapy experiences a resurgence of use for hospitalized patients, it is appropriate to consider the competencies needed by practitioners to practice safely and effectively in the inpatient setting. Hospitals differ vastly from other massage practice locations such as private offices, spas, and sports clubs. The variety of conditions encountered in an acute care setting requires knowledge and ability to adapt massage protocols appropriately.

Objectives: The Academic Collaborative for Integrative Health (ACIH) created the Hospital Based Massage Therapy (HBMT) Task Force to determine if there is a need for HBMT-specific competencies and then, if needed, develop peer-reviewed competencies that hospital staff, massage therapy educators, and massage therapists all may find useful.

Methods: The members of the Task Force identified massage therapists who worked in hospitals, as well as hospitals known to have HBMT programs, and a survey was distributed to those on the spreadsheet created. These individuals were also asked to identify others who might be interested in participating in the project. The purpose of the survey was to assess various elements of HBMT programs such as educational/experience requirements, employment model, orientation, and supervision.

Results: Thirty-two out of 37 hospitals (87\%) completed the survey. The Task Force considered the high response rate and the extent to which respondents provided in-depth information to the open-ended questions as evidence of the need for specific competencies for safe and effective massage therapy for hospitalized patients. In addition to the survey, the Task Force used a Delphi technique to engage survey participants and other experts in the field to shape the initial draft of the competencies.

Deliverables: As these competencies are shared with hospitals, massage therapists, and massage educators, the Task Force members expect that additional development of the competencies will take place as various groups implement them.

\section{MASSAGE THERAPY STUDENTS' PERCEPTIONS OF THE USE OF THE DEFENSE AND VETERANS PAIN RATING SCALE TO MONITOR CLIENT PAIN}

Julia Dais, $\mathrm{PhD}$

Introduction: The Defense and Veterans PainRating Scale (DVPRS) ${ }^{\circledR}$ is a validated, self-reporting pain assessment which includes functional language for each number on the basic $0-10$ pain scale. This along with visual cues, such as colors and faces, helps clients determine their pain scores more accurately. Supplemental questions are also included involving the impact pain has on a client's sleep, mood, stress, and activities. Change from long-standing assessment practices can be slow, but introducing the DVPRS ${ }^{\circledR}$ to student massage therapists and surveying them on their experiences is one approach.

Objectives: To determine the ease of use of the DVPRS ${ }^{\circledR}$ pain-rating scale by student massage therapists and their clients, and to determine if the DVPRS $^{\circledR}$ is helpful for initiating discussions regarding client pain patterns over time.

Methods: Two massage therapy colleges, (one in Vernon, British Columbia and one in Invercargill, New Zealand) were asked to incorporate the scale in their in student clinics and outreach programs. After 3 months of use, the student therapists were surveyed.

Results: The student massage therapists surveyed reported that they and their clients liked the features of the DVPRS ${ }^{\circledR}$ (descriptions for each pain score, the colors, and the faces), that it improved communication about their pain, and allowed for tracking of pain patterns. Many student therapists didn't always use the DVPRS ${ }^{\circledR}$ due to time constraints. However, all of the students who did use this tool recommend it to other massage therapists.

Conclusion: The DVPRS ${ }^{\circledR}$ is a validated painrating tool which helps clients chose a pain-rating score, and communicate the effect pain has on their lives, and allows for tracking of pain patterns over time. Positive responses from these students can then be used to inform practicing massage therapists of the benefits of the DVPRS ${ }^{\circledR}$ and possibly effect change across the profession. 
EFFECTS OF MASSAGE THERAPY IN PEDIATRIC PATIENTS UNDERGOING HEMATOPOIETIC STEM CELL TRANSPLANTATION: A CASE REPORT

\section{Corrie Frey, LMT, CIMI, CPMT}

Introduction: The Massage Therapy program at Nationwide Children's Hospital has developed an innovative index scale to assess efficacy of massage for the pediatric oncology patient population receiving hematopoietic cell transplantation (HCT).

Objective: The purpose of this case report is to describe how the HCT index is used to objectively record the trend of massage therapy domains over the course of the HCT process. The index addresses the following 5 domains: patient willingness to engage in therapy; orientation; pain symptoms/severity; pressure considerations; muscle tension/texture.

Case Presentation: 7 y.o. female with complex medical history, including acute lymphoblastic leukemia, underwent maternal haploidentical peripheral blood stem cell transplant. Patient received 21 days of massage therapy during a 33-day admission.

Intervention: The massage therapy evaluation occurred Day 8 prior to transplant, and treatment consisted of 1-2 sessions per day, 4-5 days per week for 30-60 minutes. The index was recorded pre- and post-treatment at evaluation and all treatment sessions. Each domain is rated on a $0-5$ point scale, with 5 being baseline values.

Interpretation: The results of the index score were U-shaped for this case study with a return to baseline levels for domains at time of discharge. Throughout the HCT process, the patient's index declined, specifically in the muscle tension/texture and pressure consideration domains.

Implications: The use of this innovative index captures the progression of the tolerance to each massage domain throughout the HCT process. The implication based on this case shows the value of tracking a patient's progress and could be used with other patient populations. We will have broad descriptive data available to report on the index, showing trends with this specific population as a part of a quality improvement project.

\section{"HANDS-ON" PALLIATIVE CARE: INTEGRATING MASSAGE THERAPY FOR PALLIATIVE CARE PATIENTS IN PAIN}

Anne Kelemen, LICSW, Hunter Groninger, MD, Lauren Cates, LMT, Mihri Mete, PhD, Gianna Shipp, Kathryn A. Walker, PharmD

Introduction: Massage therapy (MT) is the most commonly offered nonpharmacologic option in palliative care (PC) settings outside the hospital. We created an integrated approach to provide MT services to hospitalized PC patients in pain at MedStar Washington Hospital Center.

Methods: Feasibility study to measure the impact of MT on pain, medication use, and satisfaction. This randomized controlled pilot study identified a convenience sample of palliative care patients daily at MedStar Washington Hospital Center. Inclusion criteria: hospitalized adult patients receiving a palliative care consult; use of 2 or more PRN opioids in the last 24-hours; expectation of a hospital length of stay of 3 or more days. Randomized to: Arm 1: 20 min (MT) session; OR, Arm 2: 20 minute quiet time (QT) session and wait-listed for MT. 44 patients in the MT arm and 42 in the QT arm. Patients completed surveys before and after intervention, as well as the following day, to self-report pain, acceptability, and satisfaction. Data were also pulled through EMR extractions including medication use 48 hours prior to and after intervention, as well as nursing pain scores, to measure the impact of MT on opioids.

Results: 30\% decrease in pain in massage therapy group; both interventions were safe; $89 \%$ preference for massage therapy

Conclusions: Larger sample size/replication needed and this study lacked fidelity measures; study demonstrated a practical method of piloting the integration of MT into a busy PC service in a large teaching hospital. Based on pilot data, there appears to be high levels of satisfaction and acceptance related to both quiet time and massage. There was a significant decrease in pain scores following massage therapy; data on opioid use pending analysis.

\section{DEMOGRAPHIC AND UTILIZATION TRENDS OF A LONG-STANDING, HOSPITAL-BASED PEDIATRIC MASSAGE THERAPY PROGRAM}

Jake Jurbank, LMT, Kelly Tanner, PhD, OTR/L, BCP, Erin Gates, PT, DPT

Introduction: Massage therapy (MT) continues to expand in pediatric medical facilities throughout the US, however objective data on its reach continue to be lacking. In this study, new insights into patient demographics and service utilization will be presented from within a decade-old program.

Objectives: This study aims to provide demographic and diagnostic information about patients for whom massage therapy is consulted and/or utilized in a large, urban pediatric hospital.

Methods: Data from the previous year (2017) were retrospectively analyzed using descriptive statistics as a first step towards exploring the data from the 7 years since implementation of the electronic health record (EHR) in this facility. Future analyses will 
include descriptive data from all 7 years, as well as appropriate parametric statistics to determine trends. Included variables will be both demographic (age, gender, race, ethnicity) and diagnostic/admissionbased (diagnoses, primary unit, unique treatments during admission).

Results: In 2017, 16764 unique patients have been identified in our data, for which 11845 total units were billed. Another 3253 units of patient/caregiver education and face-to-face evaluations were performed. The mean age at time of treatment was 10.73 years, with an interquartile range of 1.85 to 17.1 years. A total of 315 billing providers utilized MT, led by the hematology/oncology service (3076 units) and inpatient rehab (2201 units).

Conclusions: MT is a valued tool utilized by hospital-based physicians and providers in care of patients. Further analysis in the study will include increased focus on diagnoses, and will include data across time to explore larger trends. Results thus far indicate that pediatric patients who are consulted for, or receive, MT represent a wide age range and most often are those receiving care for cancer or rehabilitation. Further research is needed to continue to track trajectories across diagnoses and time.

\section{PERCEPTIONS OF RESEARCH MASSAGE THERAPISTS UTILIZING A PROTOCOL TRAINING AND ASSESSMENT MODEL IN A RANDOMIZED CONTROLLED TRIAL}

Erika R Larson, MS, LMT, Mark Hyman Rapaport, MD, Becky Kinkead, PhD, Pamela J Schettler, PhD, Margaret Sharenko, LMT, CPT

Introduction: Collaborative partnership with a traditional research institute is one method of building research capacity in the therapeutic massage and bodywork (TMB) field. The successful collaborative partnership between Emory University's School of Medicine and Atlanta School of Massage utilized a Protocol Training and Assessment Model (Model) to train massage therapists as research massage therapists (rMTs) and maintain protocol integrity throughout the randomized controlled trial, Massage for Cancer-related Fatigue (MCRF). Components of the Model include: initial training, accessible resources, retraining, and ongoing monitoring. Taking into account stakeholder goals and experiences may inform future collaborative efforts, better enabling partnership sustainability.

Objectives: Through adaptation of a survey from a study investigating rMT perception of participation in research to reflect the views of MCRF study personnel and specifically assess the Model utilized, rMT perceptions of Model usage, Model usefulness, and MCRF participation were collected.
Methods: At MCRF completion, but prior to data analysis, a twelve-question survey was electronically sent to all MCRF rMTs. A description of the Model preceded the survey questions. Responses were analyzed for content.

Results: Six rMTs responded an average of 29 days after initial request. Responses varied in length from a single word to several sentences. Categories and themes emerged through qualitative analysis. The Model was useful for rMT participation in research. Although challenges were encountered in rMT participation in research, the experience led to rMT growth that fostered desire for an expanded role. Collaborative meetings were viewed as particularly important to engaging rMTs in MCRF.

Conclusions: Experiences of rMTs utilizing the Model and participating in MCRF indicated collaboration was a meaningful experience. The Model was useful in rMT participation in research. Insight into how to better engage rMTs in traditional-style research and improve the Model may translate to improving outcome relevance for TMB.

\section{AN OBSERVATIONAL STUDY OF MASSAGE IN WOMEN VETERANS ATTENDING A WOMENS HEALTH CLINIC}

Allison Mitchinson, MPH, BCTMB, Erika L. Trumble, MPH, Carol E. Fletcher, RN, PhD

Introduction: Research has shown that massage can reduce pain and anxiety in post-surgical, palliative, and cancer patients. Gynecologic exams and procedures can be stressful and anxiety-provoking. Massage may be effective in ameliorating pain and anxiety and increasing relaxation in women visiting a Gynecology clinic.

Objective: The purpose of this observational study was to examine the relationship between massage and well-being (pain, anxiety, shortness of breath, sense of relaxation, inner peace) in women veterans receiving care at a women's health clinic at a VA hospital.

Methods: IRB approval was obtained. Women veterans were asked by clinic staff whether they would like a relaxation massage as part of their visit. Massages were scheduled for 30-60 minutes depending on appointment schedules and given by licensed volunteer massage therapists. Effleurage massages were provided to the back, neck, hands/arms, legs/feet in a private space. Women rated their pain intensity, pain unpleasantness, anxiety, shortness of breath, relaxation, and sense of peace pre- and post-massage on a $0-10$ numeric rating scale. Demographics and data on presence of chronic pain, opioid use, and mental health diagnoses were abstracted from the medical record. 
Results: Results are based upon the first massage received by 96 unique individuals. Fifty-three percent were 40 years old or younger, $63 \%$ were Caucasian, 32\% married. Sixty-four percent were scheduled for an invasive procedure, e.g. pelvic exam, biopsy. Seventy-six percent had chronic pain, $53 \%$ had a diagnosis of depression. Mean scores for pain intensity decreased by 1.88 points, pain unpleasantness 1.99 , and anxiety by 2.42 points. Relaxation increased by 4.17 points and sense of peace by 3.57 .

Conclusion: Massage may help to reduce pain and anxiety in women veterans coming for a gynecologic health appointment. Further research should be done on the benefits of massage in this population.

\section{CLINICAL RECORD KEEPING QUALITY INITIATIVE FOR MASSAGE THERAPISTS}

\section{Steven Sebers, DC, FACO}

Introduction: The increasing integration of licensed massage therapists into the health-care system as the evidence of effectiveness for many health conditions continues to grow requires the component of high-quality clinical record keeping. Documentation of the clinical encounter using best practice guidelines is essential to support highquality patient care. Since its inception nearly 30 years ago, The CHP Group has engaged network providers in developing best practices in clinical record keeping. This has been supported by a formal clinical record quality program. This program has been well-established for naturopathic and chiropractic physicians, as well as licensed acupuncturists, and was expanded to include licensed massage therapists for this clinical quality improvement initiative (CQII).

Objectives: To develop and implement a clinical record keeping quality program specific to massage therapy.

Methods: Using an evidence-based scoring tool, 530 licensed massage therapists' clinical records were scored for quality. A threshold score was established for enrollment in an intervention group. The intervention group was given a quality improvement guide composed of best practices for clinical record keeping. After reading the guide, providers were instructed to make changes in their record-keeping procedures and submit another set of records for scoring purposes.

Results: The primary outcomes were the number of providers in the intervention group whose score improved, the degree of improvement, and the number meeting or exceeding the threshold. Of the 46 providers in the intervention group, $85 \%$ scored higher, $7 \%$ scored the same, and $9 \%$ scored lower.
Conclusion: This evidence demonstrates that an intervention in the form of a clinical record keeping quality program specific to massage therapy provided significant improvements in the evidence-based measures of clinical record keeping and supports this essential component of high-value patient care.

\section{MASSAGE AND PHYSICAL THERAPY: A COLLABORATIVE APPROACH TO PATIENT CARE IN A PEDIATRIC ONCOLOGY CLINIC}

Tiffany Silliman Cohen, LMT, CPMTII, CIMT, Stacey DiBiaso Caviston, PT, DPT, PCS, Maria R. Mascarenhas, MBBS

Introduction: Nonpharmacology-based pain management offerings, including massage and physical therapy, are an important growing component of integrative care models for pediatric patients with a cancer diagnosis.

Objectives: To work in a collaborative, interdisciplinary manner to address the physical functioning and pain management needs of pediatrics patients receiving medical treatment in oncology clinic.

Methods: In September 2018, massage therapy was initiated in the pediatric oncology clinic at our institution. Weekly work included attending morning care-coordination huddle with the medical, support, and psychosocial teams. Certified Pediatric Massage Therapist (CPMT) collaborated closely with the PT for patients who would benefit from both services to optimize their pain control, muscle balance, and physical functioning.

Results: In the first 4 months, CPMT and PT collaborated to provide ongoing support to 5 individual patients in the oncology clinic. Needs addressed included body pain/discomfort, tightness, and anxiety. After introduction of roles to the family, each provider completed their individual assessment and interventions for the patient. Providers utilized the clinic structure and flow of patient care to prioritize collaborative discussions before, between, after individual sessions. Goals of each provider were appropriately carried over in each session, and home programs were established to be complementary and sometimes in conjunction.

Conclusion: This collaborative approach in the clinic was well-received by patients, caregivers, and the interdisciplinary team. Maintaining flexibility, ongoing communications, and respecting clinic flow and hierarchy were key. Having access to both PT and CPMT on the same day and location where patients were receiving treatment positively impacted patients' abilities to participate in both services and limited the number of appointments. This improved quality of life for the patients who benefited from the services (decreased pain, improved flexibility, improved coping abilities, etc.), as well as for their 
caregivers (decreased days off work for appointments, improved care tools).

\section{PEDIATRIC MASSAGE THERAPY IN ONCOLOGY CLINIC: LESSONS LEARNED WHILE INTEGRATING INTO A PRE-EXISTING CLINIC SETTING}

Tiffany Silliman Cohen, LMT, CPMTII, CIMT, Stacey DiBiaso Caviston, PT, DPT, PCS, Maria R. Mascarenhas, MBBS

Introduction: Nonpharmacologic pain management, including massage and physical therapy, is an important, growing component of integrative care models for pediatric oncology patients.

Objectives: To improve functional status and quality of life for pediatric oncology patients by: 1) developing and integrating massage therapy with physical therapy into standard treatment; and 2) providing patients and their family with tools to reduce dysfunction, pain, and anxiety.

Methods: In September 2018, massage therapy was integrated in the pediatric oncology clinic at a tertiary care center for a half day a week. A Certified Pediatric Massage Therapist (CPMT) attended morning care-coordination huddles with the medical staff and partnered with the psychosocial team for patient selection, coordination of patient and family goals for function and connection using pediatric massage. The CPMT collaborated with the physical therapist to identify patients who would benefit from both services to optimize their pain control, muscle balance, and physical functioning.

Preliminary Results: A total of 27 patients, 25 months-24 years of age, were seen over an 11-week period, with 3-5 patient visits per session. Reasons for massage included body pain/discomfort, muscular tightness, anxiety (treatment and post-procedure), relaxation and supportive care. Interventions included: massage, caregiver education, and discussion to establish rapport. Feedback was verbally elicited post-encounter. There was consistent and unanimous positive feedback from the interdisciplinary team and patients, with no adverse outcomes noted.

Conclusion: This integrative clinic model of service delivery has shown that it is feasible to add pediatric massage therapy services into an outpatient oncology clinic. This has resulted in great inter-professional collaborative opportunities and integrated patient care that has been well received by patients, families, and the medical team.

\section{PARTNER CHAIR MASSAGE: A PILOT STUDY OF FEASIBILITY, ACCEPTABILITY, AND EFFECT ON PERINATAL MOOD, ANXIETY, AND PAIN}

\author{
Robin Byler Thomas, LMT, DrPH
}

Introduction: Perinatal mood and anxiety affects approximately $20 \%$ of women worldwide, contributing to pregnancy complications, maternal and infant bonding, and childhood development. Women seek massage for relaxation, emotional support, and pain management, but may be limited by socio-economic constraints. In prior studies, partners of pregnant women were taught a side-lying massage with demonstrated health improvements.

Objectives: Examine the feasibility, acceptability, and effects of a partner chair massage on primary outcomes to perinatal mood and anxiety, and secondary outcomes of pain.

Methods: Partners of pregnant women were taught a 10-minute chair massage and instructed to provide at home, bi-weekly for 8 weeks. Validated questionnaires measured pre-post massage effects to perinatal mood, anxiety, and pain. Weekly text messaging tracked massage dose and frequency over 8 weeks. A project-specific questionnaire evaluated feasibility and acceptability. Descriptive statistics described baseline participant data results, project specific questionnaire responses, and chair massage frequency and dose. Paired-sample $t$ tests analyzed the significance of partner chair massage effects to perinatal mood, anxiety, and pain.

Results: Twelve pregnant women (aged 32 years $+3.86 \mathrm{SD}$ ) and their partners from Tucson, AZ completed the study. Rates of retention (86\%) and protocol compliance (94\%) were high, with couples averaging 10-minute, bi-weekly chair massage throughout eight weeks. The couples reported satisfaction with study participation (97\%) and agreed chair massage was a valuable and practical skill to have in managing perinatal health symptoms $(100 \%)$. The women perceived partner chair massage as most beneficial for symptoms of perinatal mood and anxiety (92\%), and partners reported the protocol was easier to learn $(92 \%)$ than apply $(75 \%)$. Analyses of results indicated statistically significant improvements to perinatal mood $(p=.012)$ and anxiety $(p=.004)$, while a trend was observed for pain (.071).

Conclusion: This study provides the first evidence of partner chair massage as safe and effective home management of perinatal mood, anxiety, and pain. 


\section{THE VALUE OF MEDICAL MASSAGE IN NEUROLOGICAL REHABILITATION}

Inge Van Trigt, BA, MA, MMBF, Natural Therapist

Introduction: The results of 2 questionnaires conducted after the completion of two medical massage internships at the Institute of Lavigny, the neurological rehabilitation center in Lavigny, Switzerland, Europe, allowed the center to create a $0.8 \mathrm{FTE}$ medical massage position while continuing the internship position. This research conducted in 2009 and 2018 examined the added value of medical massage as perceived by (para)-medical professionals.

Objectives: To get an understanding of how (para)-medical professionals working with patients in neurorehabilitation view the added value of medical massage towards their patients, their profession, and the overall outcome of the therapeutic approach towards the patient.

Methods: 70, 8-question surveys were distributed to the (para)-medical staff. The medical massage treatments included manual lymphatic drainage, lymphatic bandaging, classical massage, connective tissue massage, and plantar reflexology. Patients were selected for treatment by the neurologist, and sessions lasted 60 minutes, 1-2 times per week for 2-20 weeks.

Results: The response rate was 49\% in 2009 and $37 \%$ in 2018 . All respondents worked with the patients who received medical massage and expressed more than $60 \%$ positivity towards the physical and psychological effects of the treatments. More than $80 \%$ of all respondents found that medical massage should become an integrated part neurological rehabilitation. The positive findings included reduction in pain medication and edema, improved range of motion, muscle and mental relaxation, as well as mood improvement. The negative findings concerned tiredness, scheduling conflicts, questionable outcomes, and concern about alleviation of a physical therapy position.

Conclusion: Medical massage is seen as an added value to the neurological rehabilitation program by the (para)-medical staff of the Institute of Lavigny and a position has been created per September, 2019. 